\title{
Research on Blade Spin - type Pipe Mixer Design and PDPA Experiment
}

\author{
Yanglin $\mathrm{Li}^{1}$, Xun Zhang ${ }^{1}$, Chundu Wu ${ }^{2}$ \\ ${ }^{1}$ Taizhou Polytechnic College, \\ ${ }^{2}$ School of Environment, Jiangsu University
}

Keywords: Pipe Mixers, Blades, PDPA, Plant Protection Machinery

\begin{abstract}
According to the requirements of on-line mixing, a new type of blade-type spin-type mixer was designed, and the PDPA technique was used to experiment with the experiment. The experiment shows that the blade spin-type mixer has the advantages of small volume, simple structure, loss of small, easy to use and maintenance, mixed effect is good, in line with online mixed use requirements.
\end{abstract}

\section{Introduction}

Mixing with the jet mixing device, the liquid is sucked into the pipeline, usually need to be mixed through the mixing tube, the simulation study, the mixing tube due to the smaller diameter, the pressure loss is large, the highest when the $30 \sim 40 \%$ the mixing uniformity of the medium (usually water) in the pipe is not ideal. The simulation results show that this phenomenon is still outstanding for emulsions. In practice, there have been mixed due to the mixing process is not uniform, different spray nozzles at the same time the liquid side of the drug injury, while the drug is not enough to reach the control effect of the phenomenon [1].

According to this situation, a blade spin type pipe mixer was designed as an auxiliary mixing device. Its structure is shown in Figure 1. The main working part is the rotor in the pipeline, the inside with the axis line (inner circle) phase tilt of the blade, the blade installed in the rotor inside the open chute (made by the line cutting) and fixed with a ride screw, the rotor Supported by axial balls, the two shells are connected by pipe threads, and both ends of the housing are connected to the pipe. The inner diameter of the rotor is the same as the pipe diameter.

At work, the fluid flows at a certain speed through the blade spin-type pipe mixer, because the blade has a certain angle, under the action of dynamic pressure, the blade automatically rotate, so that the inhalation of the liquid in both circumferential and radial direction Movement, so as to achieve the purpose of mixing evenly.

Pipeline mixers are more common in chemical and environmental engineering. There are two main categories of static mixers and dynamic mixers. The static mixer has no motive and moving parts, and the mixed liquid or gas passes through a plurality of curved runners in the static mixer to change its trajectory to achieve the purpose of mixing with each other. The dynamic mixer is the use of power to drive the mixing components, stirring the flow field, so that two different substances for forced mixing.

Chemical used in the mixer, the study is more adequate, the volume is also larger, from the relevant research can be seen, the use of power to mix, complex structure, the need for complex design of the device. It violated the original intention of online mixing design. 


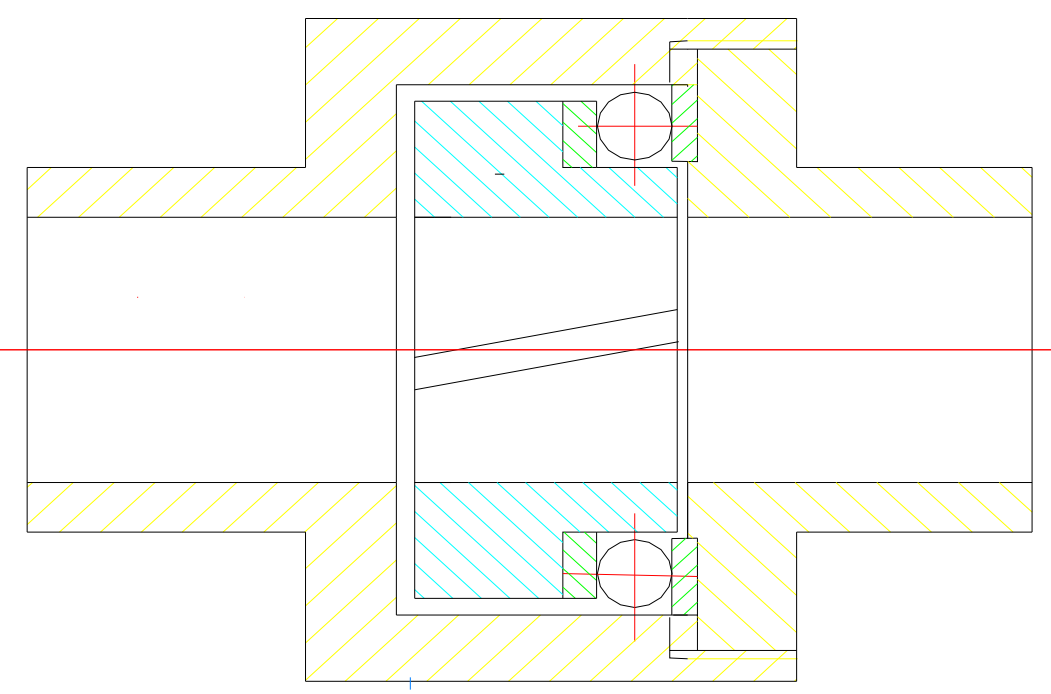

Fig.1 The structure of on line pipeline mixer

1 - imported 2 - left housing 3 - blade 4 - rotor 5 - inner ring 6 - ball (with support) 7 - outer ring 8 - right housing 9 - outlet

The experimental method, the theoretical calculation method and the numerical simulation method are used. For the experimental study, because of the large number of input and output parameters, the workload is large, Theoretically to determine the mixing time in a certain mixing time, should be calculated or measured at all times the mixer outlet cross-section of the uniformity of the components, generally need to use tracer particle photographic method [3], this method complex equipment, Data processing speed is slow, not suitable for continuous online measurement, the effect of the study mixer is not a more practical method.

With the development of fluid measurement technology, advanced laser measuring instruments such as laser Doppler Velocimetry (LDV), Particle Image Velocimetry (P IV), Phase Doppler Particle Velocimetry (PDPA) are used in rotating machine internal flow Field studies, but mostly focused on single-phase flow field measurements [4-7].

PDPA (Phase Doppler Particle Analyzer) technology is a modern advanced flow field non-contact speed technology. The technology has non-contact, fast dynamic response, wide speed range (speed ratio 100: 1), high spatial resolution, short measurement time (the shortest time of single point measurement is $0.19 \mathrm{~s}$ ), high precision measurement, real-time measurement, Space three-dimensional direction measurement and so on. [4-7] 


\section{PDPA Experimental Materials and Methods}

Measuring Device. The measuring device is shown in Figure 2:

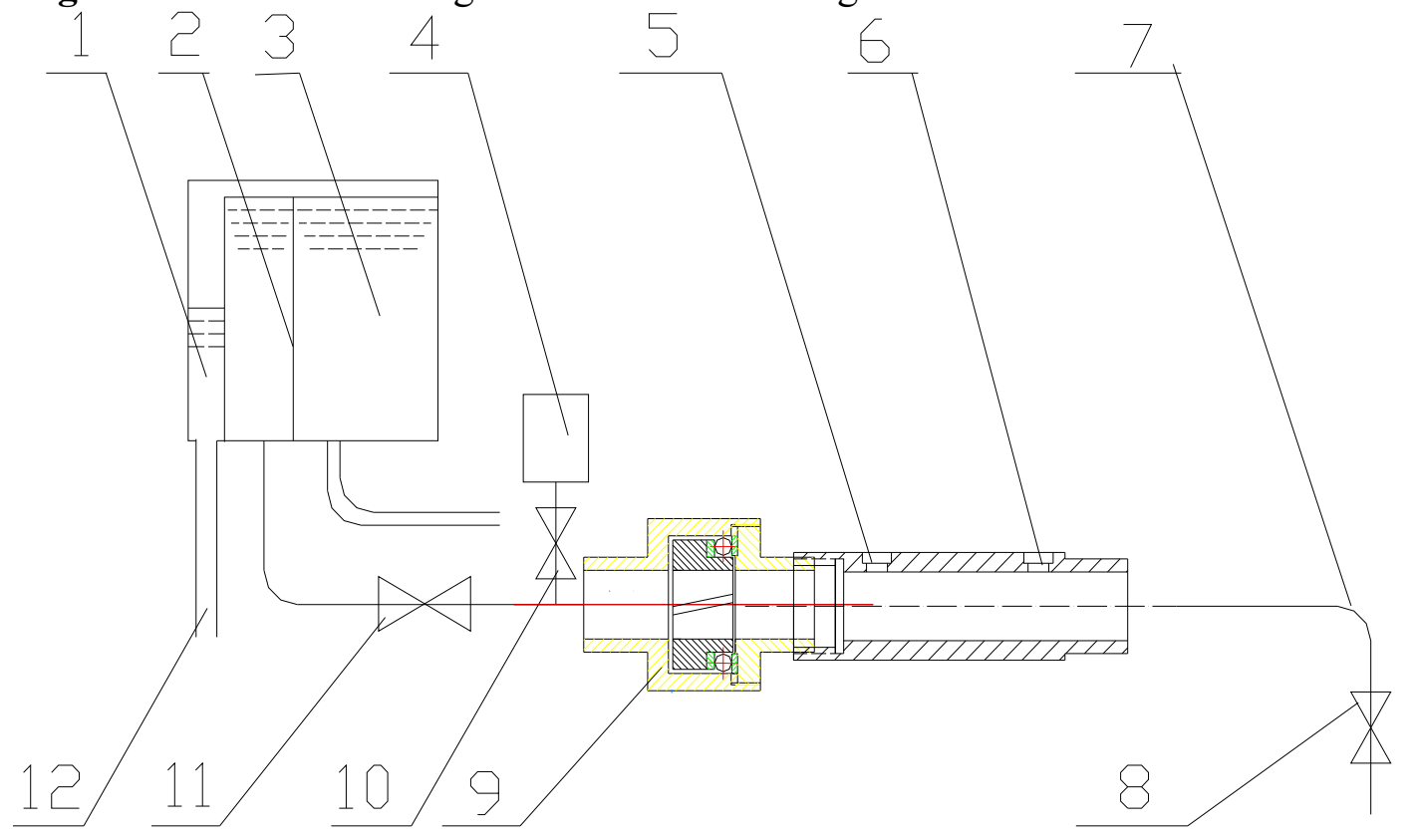

Fig. 2 Diagram of the concentration measurement PDPA equipment

1 - overflow tank 2 - inlet tank 3 - water tank 4 - mixture 5 - laser window A 16 - laser window B 27 - outlet pipe 8 - flow control valve 9 - mixer 10 - material control valve 11 - inlet valve 12 overflow pipe

The measuring device consists of three parts. The first part is the composition of water supply and dosing. Part of the water supply can ensure uniformity of pressure, divided into a sink. 3 for the water tank, through the hole with the inlet tank into the 2 into the sink, the water reaches the top of a certain height into the overflow tank, through the pipeline 12 out. This ensures the accuracy and stability of the inlet pressure. It enters the mixer 9 through the valve 11 while simulating the liquid (tracer particles) also enters the mixer at the front end of the mixer.

Mixing the mixture into the process after the flow of transparent transparent plexiglass, transparent plexiglass in the 5, 6 position of the crystal glass laser entrance window, the laser into the flow field, reflected by the particles and plexiglass reflection into the receiving probe.

\section{PDPA Experimental Results and Analysis}

The measurement results are shown in Figure 3 for the specific data measured for the PDPA system. The flow rate is $6 \mathrm{~L} / \mathrm{min}$ and the flow rate is $10 \mathrm{~L} / \mathrm{min}$.

Figure 3 shows the number of particles flowing through the unit volume of different positions on a diameter measured by window A. The figure below shows the number of particles flowing in different positions per unit diameter measured at a certain diameter.

The measured blade angle is 5 degrees. 


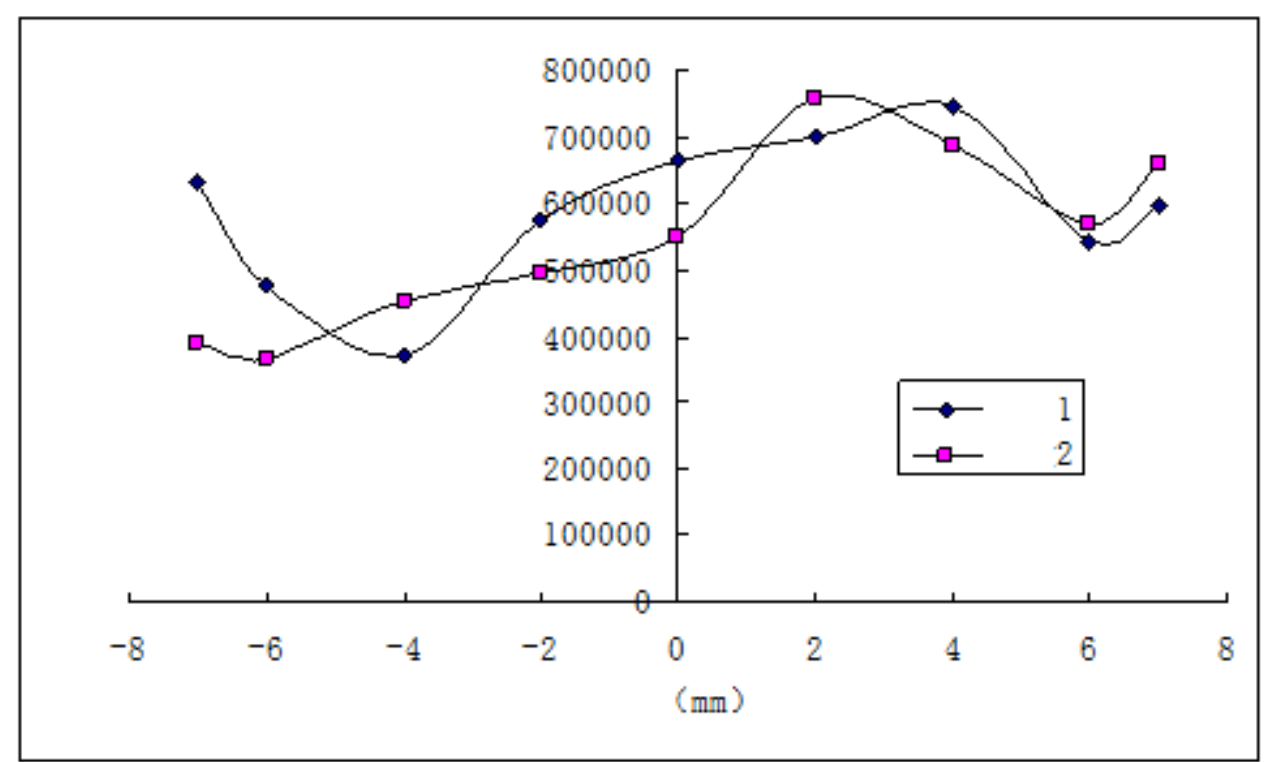

Measure window A

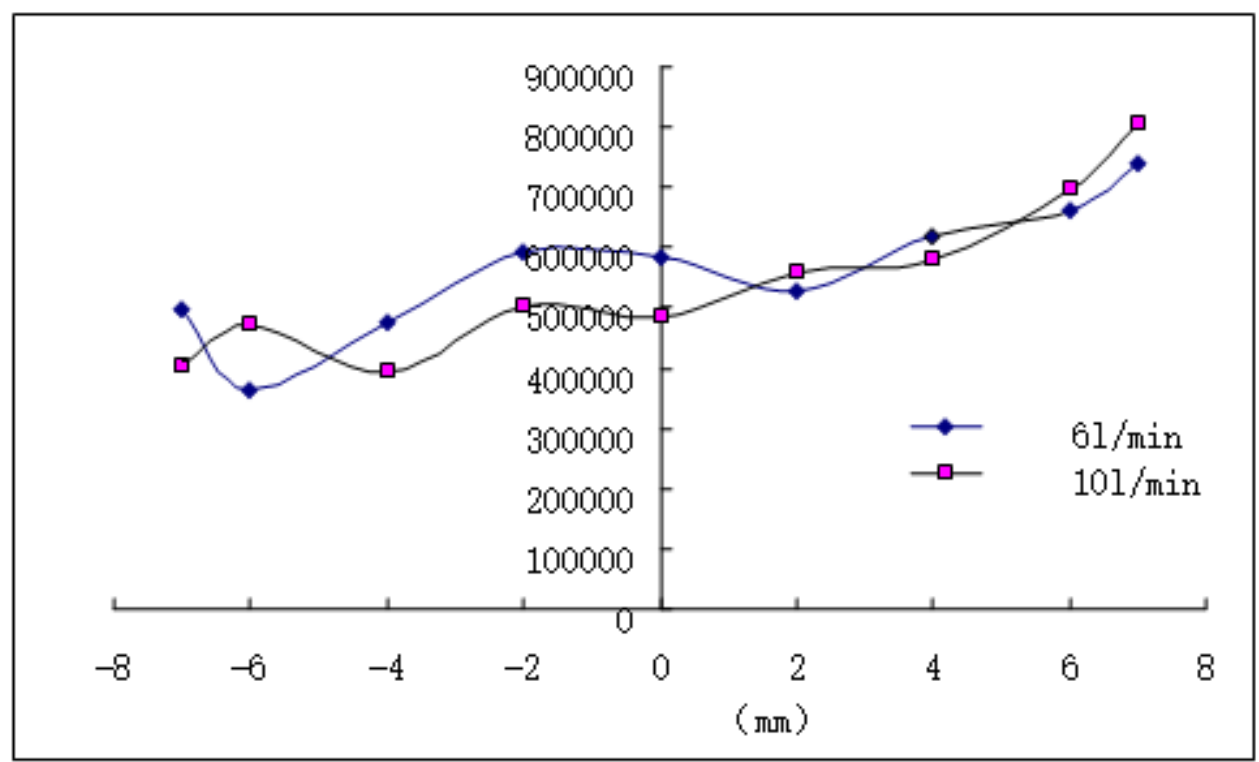

Measure window B

Fig. 3 PDPA result of the number of particles flowing through per unit

The determination of the mixing degree can be determined by the formula 1 , but since the average volume concentration cannot be determined, the following formula is used to obtain the approximate average concentration:

$$
\bar{P}=\frac{\sum_{i=1}^{n} P_{i}}{n}
$$

Where $\mathrm{n}$ is the number of points to be measured and Pi is the number of particles to be sampled. The mixing degree is calculated as follows:

Tab. 1 Mixing degree calculated from experiment results

\begin{tabular}{|l|l|l|}
\hline & PositionA & PositionB \\
\hline Flow $1(6 \mathrm{~L} / \mathrm{min})$ & 0.920 & 0.9153 \\
\hline Flow $2(10 \mathrm{~L} / \mathrm{min})$ & 0.941 & 0.937 \\
\hline
\end{tabular}

As can be seen from the above table, the evaluation of the mixing effect of the mixer reached a better grade. 
As the plexiglass can do the length is limited, the relative position of the two windows only $5 \mathrm{~cm}$, so the mixing effect did not change significantly.

In the case of different flow conditions, the mixing degree is obviously different. As the flow rate increases, the mixing degree is increased. This is because the flow rate increases, the rotation speed of the blade increases, the internal flow field changes drastically and the mixing effect increases reason.

\section{Conclusion}

(1) PDPA (phase Doppler particle analyzer) technology is a modern advanced flow field non-contact speed technology. The principle of measurement is introduced, and the analysis of the principle according to its principle is carried out to determine the number of units with the number of particles as the measured value.

(2) The mixing of different flow and position mixers was measured and the corresponding conclusions were drawn.

(3) The purpose of the new mixer is to provide a reference for the structural design and process design of the mixer.

\section{References}

[1] Wu Jia: Summary of flow measurement methods and the latest advances in the instrument, measurement technology, 2008, Volume 25, No. 6 1-4

[2] Yang Minguan Gao Bo Liu Dong Li Hui Gu Haifei: PDPA Measurement and Analysis of Liquid-Solid Two-Phase Flow Field in Swirl Pumps, Journal of Agricultural Mechanics, Vol. 38 No. $12,53-5$

[3] Shen Xiong Dong Peng Chen Qiaoning Yu Jinsheng Zhang Song: Application of dual lens three-dimensional LDV ö PDPA system to diagnose flow characteristics of fluid flow method, fluid mechanics experiment and measurement, Volume 12, No. 4, 2007

[4] LIU Zhao-hui ZHENG Chu-guang WANG Han-fang: PDPA experimental study of turbulent gas-particle two-phase flow under different swirl numbers, Journal of Chemical Industry, Vol. 52, No. 6, June 2001

[5] Qian Guihua, Sun Guogang, Li Jinghai: Effect of PDPA Measurement Volume on Measurement Results, Chemical Metallurgy, vol. 17.55, 1996

[6] Fan Jiechuan: mobile display and measurement technology, aeronautical science and technology, 1995, No. 6

[7] Teng Biaoying, Li Yongguang, Zhou Weiguo, Ma Xinxia: Status and Prospect of Gas-solid Two-phase Flow Measurement Technology. Journal of Shanghai University of Electric Power. Vol. 18, No. 4 December 2002 